\title{
Facilitators and barriers to autonomy: a systematic literature review for older adults with physical impairments, living in residential care facilities
}

\author{
Jolande van Loon ${ }^{1,2,3 *}$ (D), Katrien Luijkx ${ }^{1}$, Meriam Janssen ${ }^{1}$, Ietje de Rooij ${ }^{2}$ \\ and Bienke Janssen ${ }^{3}$ \\ ${ }^{1}$ Department of Tranzo, School of Social and Behavioural Sciences, Tilburg University, Tilburg, The \\ Netherlands, ${ }^{2}$ De Wever, Tilburg, The Netherlands and ${ }^{3}$ Fontys University of Applied Sciences, Fontys \\ School of People and Health, Bachelor of Nursing and Knowledge Centre in Person-centred Practice in \\ Health and Social Care, Eindhoven, The Netherlands \\ ${ }^{*}$ Corresponding author. Email: J.M.C.vanLoon@tilburguniversity.edu
}

(Accepted 26 September 2019; first published online 30 October 2019)

\begin{abstract}
Autonomy is important in every stage of life. However, little is known about how autonomy is enhanced for older adults living in residential care facilities (RCFs). This leads to the research question: which facilitators and barriers to autonomy of older adults with physical impairments due to ageing and chronic health conditions living in RCFs are known? The results will be organised according to the framework of person-centred practice, because this is related to autonomy enhancement. To answer the research question, a systematic literature search and review was performed in the electronic databases CINAHL, PsycINFO, PubMed, Social Services Abstracts and Sociological Abstracts. Inclusion and exclusion criteria were derived from the research question. Selected articles were analysed and assessed on quality using the Mixed Methods Appraisal Tool. Facilitators and barriers for autonomy were found and arranged in four themes: characteristics of residents, prerequisites of professional care-givers, care processes between resident and care-giver, and environment of care. The established facilitators and barriers are relational and dynamic. For a better understanding of the facilitators and barriers to autonomy for older adults with physical impairments living in RCFs, a description is based on the 35 included articles. Autonomy is a capacity to influence the environment and make decisions irrespective of having executional autonomy, to live the kind of life someone desires to live in the face of diminishing social, physical and/or cognitive resources and dependency, and it develops in relationships. The results provide an actual overview and lead to a better understanding of barriers and facilitators for the autonomy of older adults with physical impairments in RCFs. For both residents and care-givers, results offer possibilities to sustain and reinforce autonomy. Scientifically, the study creates new knowledge on factors that influence autonomy, which can be used to enhance autonomy.
\end{abstract}

(C) Cambridge University Press 2019. This is an Open Access article, distributed under the terms of the Creative Commons Attribution licence (http://creativecommons.org/licenses/by/4.0/), which permits unrestricted re-use, distribution, and reproduction in any medium, provided the original work is properly cited. 
Keywords: autonomy; residents; physically impaired; residential care facilities; older adults; person-centred practice; nursing home; systematic review

\section{Introduction}

In many Western countries, governments see active citizenship as an important theme. A neoliberal philosophy has influenced and changed health-care systems during the last decade. Now, citizens should take personal responsibility and participate in society as independent individuals. This responsibility also concerns health and welfare (Verhoeven and Tonkens, 2013). The worldwide trend in health care is that older adults with chronic conditions and physical impairments continue to live at home (Cartier, 2003; Bjornsdottir, 2009; Jacobs, 2019). Policies are directed towards self-management and informal care from family and friends.

If living at home is no longer possible - e.g. due to severe physical impairments admission to a residential care facility (RCF) is permitted. How is participation achieved in a facility that is a place to live as an individual, as well as a place where the resident is dependent on others to receive appropriate care? The authors focus on older adults with psychical impairments due to age-related decline and chronic health conditions (further to be called: residents with physical impairments). Generally speaking, these persons are able to make decisions on how they want to live their lives, but are often not able to execute the decisions they make themselves. The focus of this review article is to gain insight into which facilitators and barriers influence autonomy of older adults with physical impairments.

Living in residential care influences autonomy. The authors are investigating this influence because they have the presumption that intervening on these facilitators and barriers for this specific group will create better opportunities for their autonomy.

The concept of participation is discussed in the light of diverse psychological and sociological research and is described with words such as 'control', 'agency', 'mastery', 'autonomy', 'self-management' and 'self-determination' (Morgan and Brazda, 2013). The authors of the current review chose to use the word autonomy because of the decisional versus executional polarity. This polarity was described by Collopy (1988) as follows: a resident can have a desire and make decisions on how she/he wants to live her/his life, even if she/he cannot actualise them.

Moreover, in RCFs, several residents with physical impairments live together and can simultaneously have incompatible needs and wishes (Bolmsjö et al., 2006). Autonomy is given shape in a relational context between staff and other residents (Abma et al., 2012; Baur and Abma, 2012; Gleibs et al., 2014; Oosterveld-Vlug et al., 2014). McCormack (2001) challenges the 'individualistic concept of autonomy' as used in neoliberal tradition and gives a different view based on interconnectedness and person-centred care.

The aforementioned relationship between autonomy and person-centred care can help to study autonomy in more detail. The aim of person-centred care is to place residents at the centre: in other words, each resident is seen as a unique person with a personal history, future and life goals. With person-centred care, caregivers can respect and enhance autonomy of residents in the last phase of their lives (Danhauer et al., 2006; Donnelly and MacEntee, 2016). McCormack and McCance (2017) formulated a leading theory of person-centred practice (PCP) which can 
help to reflect upon facilitators and barriers to autonomy. It offers a theoretical, evidence-based framework. PCP is seen as a multi-dimensional concept and it is still developing. It takes into account person-centred outcomes (e.g. involvement in care), person-centred processes (e.g. sharing decision-making), the care environment (e.g. appropriate skills mix in the nursing team), prerequisites of staff (e.g. providing holistic care) and the macro context (e.g. health and social care policy) (McCormack and McCance, 2017).

A better understanding of the factors that strengthen autonomy (facilitators) or undermine autonomy (barriers) can help to enhance practices in RCFs that lead to interventions to preserve and facilitate autonomy of older adults with physical impairments living in RCFs. For a better understanding, the authors will underpin the concept of autonomy for older adults living in RCFs with a description that will be derived from the literature.

A systematic literature review will be executed with the research question: which facilitators and barriers to autonomy of older adults with physical impairments due to ageing and chronic health conditions living in RCFs are known?

\section{Method}

To answer the research question, a systematic literature search was conducted in the following databases: PubMed, CINAHL, Social Services Abstracts, Sociological Abstracts and PsycINFO. These databases include articles about care, cure and psycho-social functioning. For the central aspects, living in an institution for longterm care and autonomy, the thesaurus (Social Services Abstracts, Sociological Abstracts and PsycINFO), MESH terms (PubMed) and headings (CINAHL) were used to select search terms that best matched the research question (Table 1). The search was conducted in March 2016 and updated in July 2017. A limit of ten years (beginning from 2006) was chosen, because the neoliberal approach of participation and the role of autonomy has only been put into laws and regulations over the last decade. The question of how autonomy can be enhanced for the more vulnerable members of society also emerged in this period.

Inclusion and exclusion criteria were established to be sure to review articles that concern the residents under study, namely older adults with physical impairments due to ageing and chronic conditions who live in RCFs (Table 2).

\section{Selection}

Figure 1 shows the results of the database search. Using the inclusion and exclusion criteria (Table 2), the titles of the 3,030 unique articles were screened by the first author (JvL). When in doubt, the article went to the next stage. Selection by abstract was performed independently by JvL and three co-authors (KL, IdR and BJ). These co-authors each reviewed one-third of the articles and JvL reviewed all the articles. Afterwards, the selections were discussed in pairs of reviewers in order to reach a consensus. When no consensus was reached on an article, it was included in the next stage. The same procedure was followed for the full-text selection. When no consensus about inclusion or exclusion was reached in this stage, a third author was consulted and a consensus was reached. 
Table 1. Search terms and strategy

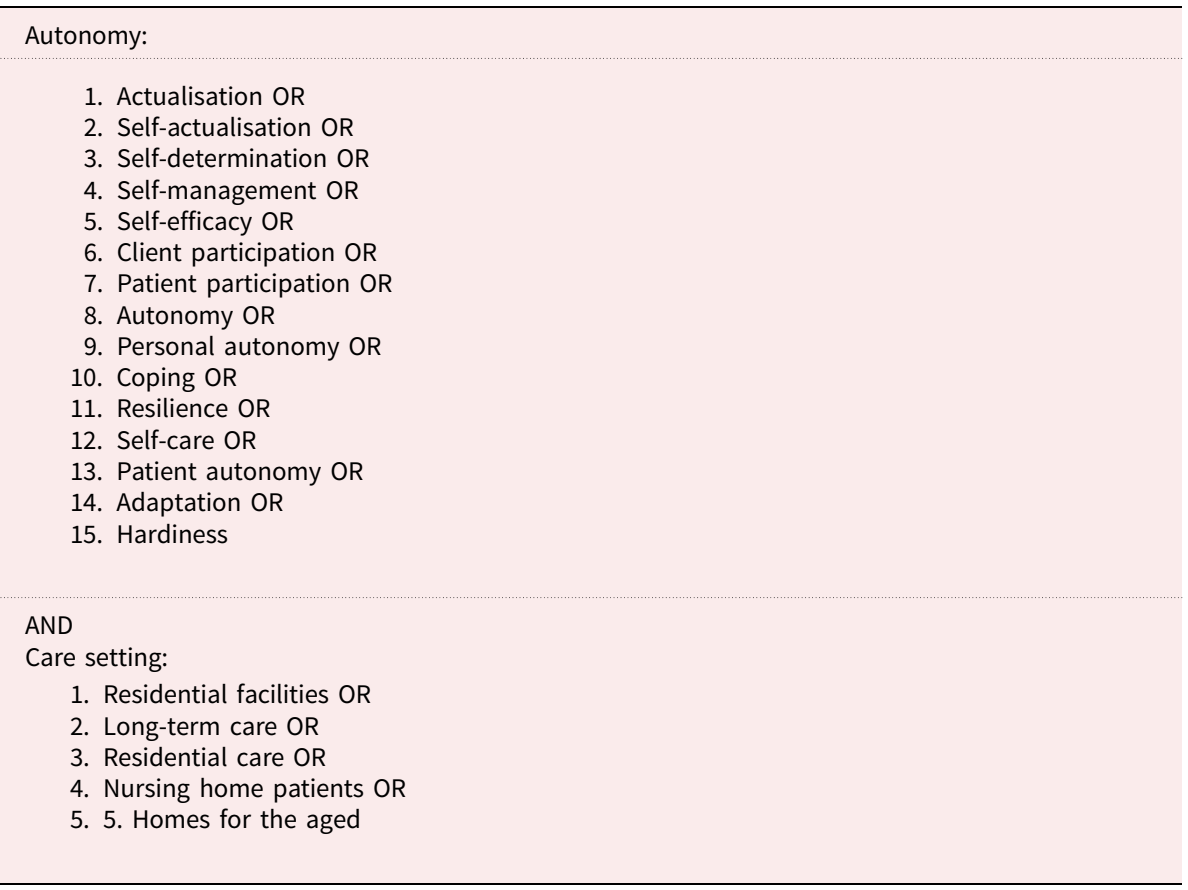

Table 2. Inclusion and exclusion criteria for autonomy and its facilitators and barriers

Inclusion:

1. Older adults living in residential care facilities with physical impairments.

2. Time period March 2006 to July 2017.

3. Written in the English language.

4. Empirical research.

5. Peer-reviewed journal article.

6. Health technology as far as it concerns the autonomy of residents.

7. Care-givers/family care as far as it concerns the autonomy of residents.

8. Professional care-giver issues as far as it concerns the autonomy of residents.

9. The decision to move, or process of moving, to long-term care.

\section{Exclusion:}

1. Persons suffering from dementia, psychiatric disorders and mentally challenged persons.

2. Place of living: in community, hospital and rest home.

3. Average age younger than 65 years.

4. Specific diseases and impairments.

5. Specific treatments of diseases.

6. Self-management of diseases like diabetes and blood pressure control. 


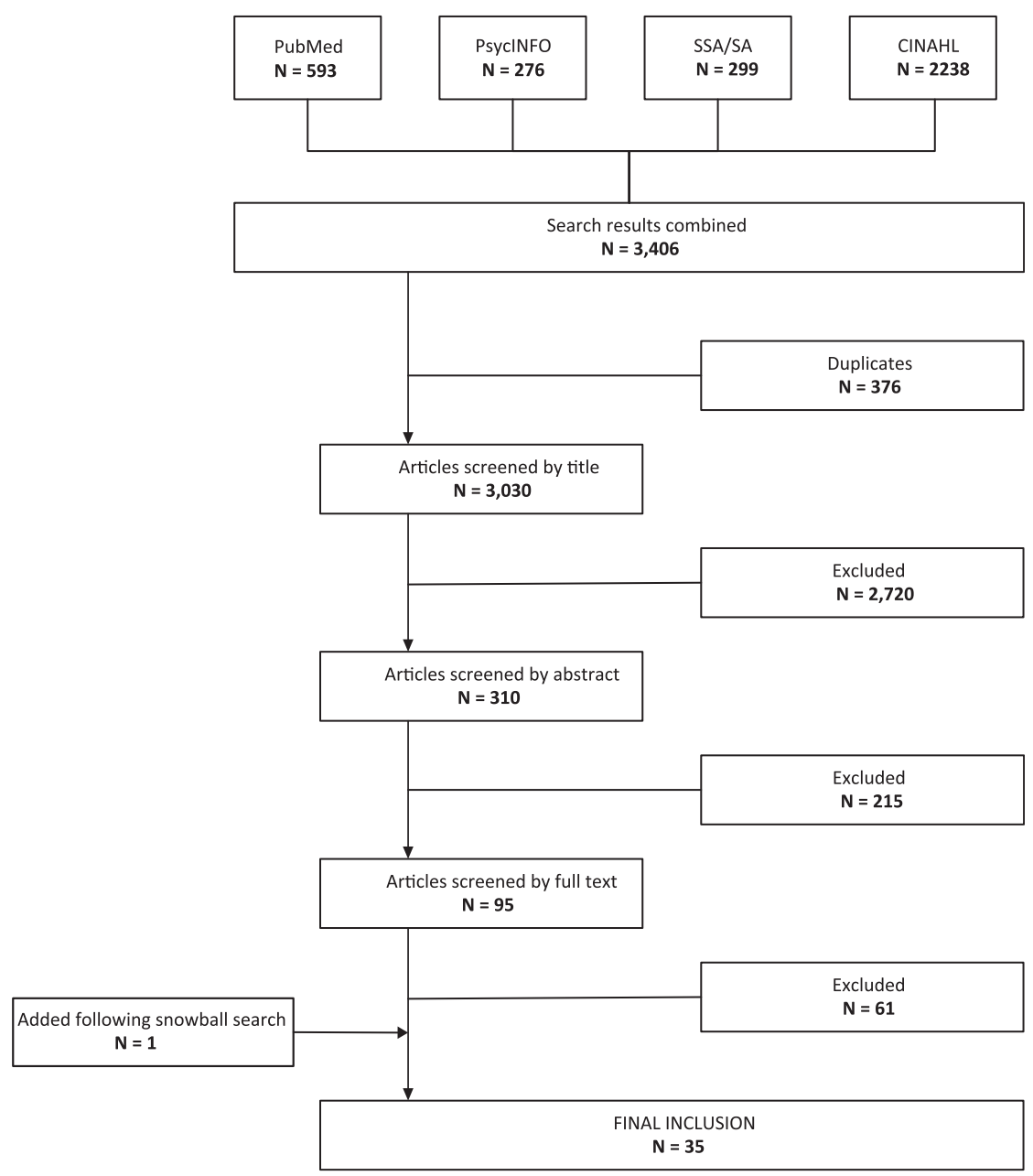

Figure 1. Flowchart of the database search of facilitators and barriers to autonomy. Note: SSA/SA: Social Services Abstracts and Sociological Abstracts.

\section{Data extraction and quality assessment of the articles}

The data extraction of the full texts was performed using a format wherein the authors independently noted the description and the position of autonomy (i.e. cause, mediator or result). Apart from one article (Brandburg et al., 2013), the descriptions were given in the Introduction section in which the authors clarify how they were going to use the concept in their study.

Subsequently, the authors noted facilitators and barriers as given in the Results sections of the articles. Afterwards JvL and KL, JvL and IdR, and JvL and BJ compared and discussed the extracted data in order to compare and interpret the data.

Each article was also assessed on quality, again independently by JvL and KL, IdR and BJ. The results of the assessed quality were also discussed in bilateral 
sessions. Because the systematic review includes articles with qualitative, quantitative and mixed-methods designs, the Mixed Methods Appraisal Tool (MMAT) was used to assess the quality of the selected articles. The MMAT is developed to facilitate the concurrent appraisal of articles with different designs, and provides elements to assess the quality of the articles to be included (Pace et al., 2012). In order to do so, four elements for studies with a qualitative or quantitative design are defined; for mixed-method designs, 11 elements are defined. The scores are reported in column 3 of Table 3.

\section{Data synthesis}

The facilitators and barriers (see Table 3, columns 7 and 8) were organised by JvL, $\mathrm{KL}$, IdR and BJ in three themes derived from the PCP framework (McCormack and McCance, 2017). Because a large group of facilitators and barriers found in the included articles concerned the residents themselves, the authors decided to add the theme 'characteristics of residents'. The current article thus uses four themes that affect autonomy, namely characteristics of residents, prerequisites of professional care-givers in RCFs, processes in the relationship between residents and professional care-givers, and the care environment. When the context of the facilitators and barriers in an included article was not clear enough to assign it to one theme, the authors chose to assign it to more than one.

The included studies did differ in method and quality. However, the authors decided not to exclude the six articles scoring below 75 per cent because they provided relevant information on the research question. Moreover, in the analyses and presentation of the results, articles with a low MMAT score will not dominate.

Elements from the descriptions (Table 3, column 6) were used to make a general description of autonomy for older adults with physical impairments in RCFs.

\section{Reliability}

The authors started with an individual review of ten abstracts using the inclusion and exclusion criteria. In a meeting, they discussed the similarities and differences in the selection. The same was done in the stage of the full-text selection, this time with two articles. In this way, a uniform selection procedure of abstracts and full texts was achieved. At each selection stage, the first author (JvL) reviewed all the articles and the co-authors (KL, IdR and BJ) each reviewed one-third of the articles. The articles were discussed bilaterally between JvL with KL, IdR and BJ. When no consensus was reached, the article was reviewed again in the next stage. At each stage, the articles switched to another reviewer. The stage of data extraction and quality assessment was also preceded by a meeting with all reviewers to discuss the analysis and assessment process.

\section{Results}

The search identified 3,030 unique articles, of which 35 were included. Table 3 (column 2) shows that most of the articles originate from North-West Europe, Australia and North America. The MMAT scores (column 3) vary from 25 to 
Table 3. Description of the included articles and results

\begin{tabular}{|c|c|c|c|c|c|c|c|}
\hline Authors and year & Country & $\begin{array}{l}\text { MMAT } \\
\text { quality } \\
\text { score } \\
(\%)\end{array}$ & Title of the article & $\begin{array}{l}\text { Design }(D), \\
\text { number of } \\
\text { participants }(N) \text {, } \\
\text { population }(P), \\
\text { age }(A) \text { and } \\
\text { mean age }(M)\end{array}$ & $\begin{array}{c}\text { Description of } \\
\text { autonomy (as } \\
\text { described in the } \\
\text { Introduction section) }\end{array}$ & $\begin{array}{l}\text { Facilitators (as } \\
\text { described in the } \\
\text { Results section) }\end{array}$ & $\begin{array}{c}\text { Barriers (as described } \\
\text { in the Results } \\
\text { section) }\end{array}$ \\
\hline Abma et al., 2012 & NL & 100 & $\begin{array}{l}\text { Responsibilities in } \\
\text { elderly care: Mr } \\
\text { Powell's narrative of } \\
\text { duty and relations }\end{array}$ & $\begin{array}{l}\text { D: Qualitative: } \\
\text { observations } \\
\text { N: } 1 \\
\text { P: Resident } \\
\text { A: } 92 \\
\text { M: Not } \\
\text { applicable }\end{array}$ & $\begin{array}{l}\text { Autonomy is relational } \\
\text { and 'entails self-worth } \\
\text { and self-development' } \\
\text { and is 'an interactive } \\
\text { process requiring the } \\
\text { help and support of } \\
\text { others' (p. 28). }\end{array}$ & $\begin{array}{l}\text { Helping to see } \\
\text { limitations. } \\
\text { Reciprocity and } \\
\text { mutuality. Listening } \\
\text { to life stories. }\end{array}$ & $\begin{array}{l}\text { Not listening. Not } \\
\text { helping to adapt } \\
\text { expectations. }\end{array}$ \\
\hline $\begin{array}{l}\text { Anderberg and } \\
\text { Berglund, } 2010\end{array}$ & Sweden & 100 & $\begin{array}{l}\text { Elderly persons' } \\
\text { experiences of } \\
\text { striving to receive } \\
\text { care on their own } \\
\text { terms in nursing } \\
\text { homes }\end{array}$ & $\begin{array}{l}\text { D: Qualitative: } \\
\text { interviews } \\
\text { N: } 15 \\
\text { P: Residents } \\
\text { A: } 73-98 \\
\text { M: } 82.4\end{array}$ & $\begin{array}{l}\text { Maintaining the } \\
\text { 'abilities in order to } \\
\text { have a sense of } \\
\text { control in their life' } \\
\text { (p. 67). }\end{array}$ & $\begin{array}{l}\text { Learning dimension: } \\
\text { the ability to find } \\
\text { your way in the } \mathrm{NH} \text {. }\end{array}$ & \\
\hline Andresen et al., 2009 & Denmark & 50 & $\begin{array}{l}\text { Perceived autonomy } \\
\text { and activity choices } \\
\text { among physically } \\
\text { disabled older } \\
\text { people in nursing } \\
\text { home settings: a } \\
\text { randomized trial }\end{array}$ & $\begin{array}{l}\text { D: Quantitative: } \\
\text { questionnaires } \\
\text { N: } 50 \\
\text { P: Residents } \\
\text { A: } 65-97 \\
\text { M: - }\end{array}$ & $\begin{array}{l}\text { Perceived autonomy } \\
\text { in choice and control } \\
\text { over activities in daily } \\
\text { life. }\end{array}$ & $\begin{array}{l}\text { Individually tailored } \\
\text { programmes for } \\
\text { activities. }\end{array}$ & $\begin{array}{l}\text { Schedules for } \\
\text { activities such as rest. }\end{array}$ \\
\hline Baur and Abma, 2012 & $\mathrm{NL}$ & 100 & $\begin{array}{l}\text { 'The Taste Buddies': } \\
\text { participation and } \\
\text { empowerment in a } \\
\text { residential home for } \\
\text { older people }\end{array}$ & $\begin{array}{l}\text { D: Qualitative: } \\
\text { action research } \\
\text { N: } 7 \\
\text { P: Residents } \\
\text { A: } 82-92 \\
\text { M: - }\end{array}$ & $\begin{array}{l}\text { Empowerment } \\
\text { through collective } \\
\text { participation. }\end{array}$ & $\begin{array}{l}\text { Relational process } \\
\text { as a catalyst for } \\
\text { change. Supporting } \\
\text { environment; role } \\
\text { models facilitate } \\
\text { empowerment. }\end{array}$ & \\
\hline
\end{tabular}


Table 3. (Continued.)

\begin{tabular}{|c|c|c|c|c|c|c|c|}
\hline Authors and year & Country & $\begin{array}{l}\text { MMAT } \\
\text { quality } \\
\text { score } \\
(\%)\end{array}$ & Title of the article & $\begin{array}{l}\text { Design }(D), \\
\text { number of } \\
\text { participants }(N) \text {, } \\
\text { population }(P), \\
\text { age }(A) \text { and } \\
\text { mean age }(M)\end{array}$ & $\begin{array}{l}\text { Description of } \\
\text { autonomy (as } \\
\text { described in the } \\
\text { Introduction section) }\end{array}$ & $\begin{array}{l}\text { Facilitators (as } \\
\text { described in the } \\
\text { Results section) }\end{array}$ & $\begin{array}{c}\text { Barriers (as described } \\
\text { in the Results } \\
\text { section) }\end{array}$ \\
\hline Bolmsjö et al., 2006 & Sweden & 100 & $\begin{array}{l}\text { Everyday ethics in } \\
\text { the care of elderly } \\
\text { people }\end{array}$ & $\begin{array}{l}\text { D: Qualitative: } \\
\text { observations } \\
\text { N1: } 12 \text { observed } \\
\text { P1: Residents } \\
\text { A: - } \\
\text { M: - } \\
\text { N2: - } \\
\text { P2: Staff of P1 } \\
\text { A: } 20-60 \text { years } \\
\text { M: - }\end{array}$ & $\begin{array}{l}\text { Autonomy is 'having } \\
\text { the possibility to } \\
\text { decide, influence and } \\
\text { have choice in ... daily } \\
\text { life } \\
\text { (self-determination) } \\
\text { and also by not being } \\
\text { dependent' ( } p .253 \text { ). }\end{array}$ & $\begin{array}{l}\text { Ethical competence } \\
\text { of staff leads to } \\
\text { autonomy. }\end{array}$ & $\begin{array}{l}\text { Ethical incompetence } \\
\text { leads to less } \\
\text { autonomy and } \\
\text { wellbeing. }\end{array}$ \\
\hline $\begin{array}{l}\text { Brandburg et al., } \\
2013\end{array}$ & USA & 100 & $\begin{array}{l}\text { Resident strategies } \\
\text { for making a life in a } \\
\text { nursing home: a } \\
\text { qualitative study }\end{array}$ & $\begin{array}{l}\text { D: Qualitative: } \\
\text { interviews } \\
\mathrm{N}: 21 \\
\mathrm{P}: \text { Residents } \\
\text { A: } 65-93 \\
\mathrm{M}:-\end{array}$ & $\begin{array}{l}\text { Resilience: 'ability to } \\
\text { adapt successfully to } \\
\text { challenges in life' } \\
\text { (p. 866). } .^{1}\end{array}$ & $\begin{array}{l}\text { Personal resilience } \\
\text { and strategies for } \\
\text { coping and } \\
\text { adapting (after a } \\
\text { move). }\end{array}$ & $\begin{array}{l}\text { Unplanned move to } \\
\mathrm{NH} \text { interferes with } \\
\text { successful } \\
\text { adaptation. }\end{array}$ \\
\hline Chao et al., 2008 & Taiwan & 50 & $\begin{array}{l}\text { Predictors of } \\
\text { psychosocial } \\
\text { adaptation among } \\
\text { elderly residents in } \\
\text { long-term care } \\
\text { settings }\end{array}$ & $\begin{array}{l}\text { D: Quantitative: } \\
\text { questionnaires } \\
\text { P: Residents } \\
\text { N: } 126 \\
\text { A: } 65-102 \\
\text { M: } 81\end{array}$ & $\begin{array}{l}\text { Psycho-social } \\
\text { adaptation: 'the } \\
\text { ability of elders to } \\
\text { maintain a sense of } \\
\text { self-identity and } \\
\text { continue valued roles } \\
\text { and interactions with } \\
\text { others while adapting } \\
\text { to life in a long-term } \\
\text { care facility' (p. 150). }\end{array}$ & $\begin{array}{l}\text { Voluntary } \\
\text { admission. Having } \\
\text { roommates. Higher } \\
\text { functional status. } \\
\text { Financial resources. } \\
\text { Having family } \\
\text { support. }\end{array}$ & $\begin{array}{l}\text { Absence of family } \\
\text { support. }\end{array}$ \\
\hline
\end{tabular}




\begin{tabular}{|c|c|c|c|c|c|c|c|}
\hline Cooney et al., 2009 & Ireland & 100 & $\begin{array}{l}\text { Resident } \\
\text { perspectives of the } \\
\text { determinants of } \\
\text { quality of life in } \\
\text { residential care in } \\
\text { Ireland }\end{array}$ & $\begin{array}{l}\text { D: Qualitative: } \\
\text { interviews } \\
\text { N: } 101 \\
\text { P: Residents } \\
\text { A: } 65-90+ \\
\text { M: - }\end{array}$ & $\begin{array}{l}\text { Quality of life: 'sense } \\
\text { of wellbeing, } \\
\text { perceived happiness } \\
\text { and life satisfaction ... } \\
\text { independence, social } \\
\text { activity and perceived } \\
\text { control over life' } \\
\text { (p. 1030). }\end{array}$ & $\begin{array}{l}\text { Maximising } \\
\text { potential: ethos of } \\
\text { care, sense of self } \\
\text { and identity, } \\
\text { connectedness, } \\
\text { activities and } \\
\text { therapies. Physical } \\
\text { environment: } \\
\text { homely } \\
\text { atmosphere. Social } \\
\text { environment: good } \\
\text { relationships with } \\
\text { continuity and } \\
\text { reciprocity. }\end{array}$ & $\begin{array}{l}\text { Physical } \\
\text { environment: } \\
\text { absence of homely } \\
\text { social spaces; poor } \\
\text { toilet and bathroom } \\
\text { provisions. Social } \\
\text { environment: staff } \\
\text { are seen as too busy; } \\
\text { negative social } \\
\text { relations; no } \\
\text { potential to stay } \\
\text { connected. }\end{array}$ \\
\hline Curtiss et al., 2007 & USA & 25 & $\begin{array}{l}\text { Motivation style, } \\
\text { lengths of residence, } \\
\text { voluntariness, and } \\
\text { gender influences on } \\
\text { adjustment to } \\
\text { long-term care: a } \\
\text { pilot study }\end{array}$ & $\begin{array}{l}\text { D: Quantitative: } \\
\text { questionnaires } \\
\text { N: } 75 \\
\text { P: Residents } \\
\text { A: } 69.5-88.8 \\
\text { M: - }\end{array}$ & $\begin{array}{l}\text { Self-determination } \\
\text { style that is related to } \\
\text { adaptation. }\end{array}$ & $\begin{array}{l}\text { Self-determined } \\
\text { coping style helps } \\
\text { to cope with } \\
\text { institutional life. }\end{array}$ & \\
\hline Custers et al., 2010 & NL & 75 & $\begin{array}{l}\text { Need fulfillment in } \\
\text { caring relationships: } \\
\text { its relation with } \\
\text { well-being of } \\
\text { residents in somatic } \\
\text { nursing homes }\end{array}$ & $\begin{array}{l}\text { D: Quantitative: } \\
\text { questionnaires } \\
\text { N: } 88 \\
\text { P: Residents } \\
\text { A: } 50-97 \\
\text { M: } 78.5\end{array}$ & $\begin{array}{l}\text { Autonomy: 'one can } \\
\text { choose activities, } \\
\text { make decisions and } \\
\text { regulate behaviour in } \\
\text { accordance with own } \\
\text { goals' (p. 732). }\end{array}$ & $\begin{array}{l}\text { High-quality care } \\
\text { relationships } \\
\text { contribute to need } \\
\text { fulfilment, less } \\
\text { depressive feelings } \\
\text { and more life } \\
\text { satisfaction. }\end{array}$ & \\
\hline Custers et al., 2011 & $\mathrm{NL}$ & 100 & $\begin{array}{l}\text { Need support and } \\
\text { wellbeing during } \\
\text { morning care } \\
\text { activities: an } \\
\text { observational study } \\
\text { on resident-staff } \\
\text { interaction in } \\
\text { nursing homes }\end{array}$ & $\begin{array}{l}\text { D: Quantitative: } \\
\text { observation and } \\
\text { questionnaires } \\
\text { N: } 20 \\
\text { P: Residents } \\
\text { A: } 54-93 \\
\text { M: } 79.1\end{array}$ & $\begin{array}{l}\text { Autonomy: 'one can } \\
\text { choose activities, } \\
\text { make decisions and } \\
\text { regulate behaviour in } \\
\text { accordance with own } \\
\text { goals' (p. 1428). }\end{array}$ & $\begin{array}{l}\text { Care-givers that } \\
\text { fulfil the need for } \\
\text { autonomy, } \\
\text { relatedness and } \\
\text { competence. }\end{array}$ & \\
\hline
\end{tabular}


Table 3. (Continued.)

\begin{tabular}{|c|c|c|c|c|c|c|c|}
\hline Authors and year & Country & $\begin{array}{l}\text { MMAT } \\
\text { quality } \\
\text { score } \\
(\%)\end{array}$ & Title of the article & $\begin{array}{l}\text { Design }(D), \\
\text { number of } \\
\text { participants }(N) \text {, } \\
\text { population }(P), \\
\text { age }(A) \text { and } \\
\text { mean age }(M)\end{array}$ & $\begin{array}{l}\text { Description of } \\
\text { autonomy (as } \\
\text { described in the } \\
\text { Introduction section) }\end{array}$ & $\begin{array}{l}\text { Facilitators (as } \\
\text { described in the } \\
\text { Results section) }\end{array}$ & $\begin{array}{c}\text { Barriers (as described } \\
\text { in the Results } \\
\text { section) }\end{array}$ \\
\hline Custers et al., 2012 & NL & 25 & $\begin{array}{l}\text { Relatedness, } \\
\text { autonomy, and } \\
\text { competence in the } \\
\text { caring relationship: } \\
\text { the perspective of } \\
\text { nursing home } \\
\text { residents }\end{array}$ & $\begin{array}{l}\text { D: Mixed } \\
\text { methods: } \\
\text { questionnaires } \\
\text { and interviews } \\
\text { N: } 35 \\
\text { P: Residents } \\
\text { A: } 55-93 \\
\text { M: } 79.9\end{array}$ & $\begin{array}{l}\text { Autonomy: 'one can } \\
\text { choose activities, } \\
\text { make decisions and } \\
\text { regulate behaviour in } \\
\text { accordance with one's } \\
\text { goals' (p. 320). }\end{array}$ & $\begin{array}{l}\text { Higher ADL } \\
\text { dependency. Match } \\
\text { between individual } \\
\text { preferences and } \\
\text { support. Asking for } \\
\text { preferences. } \\
\text { Facilitating } \\
\text { care-givers to act } \\
\text { upon the } \\
\text { preferences. Higher } \\
\text { education of staff. }\end{array}$ & \\
\hline Danhauer et al., 2006 & USA & 75 & $\begin{array}{l}\text { Accenting the } \\
\text { positive recent } \\
\text { 'uplifts' reported by } \\
\text { nursing home } \\
\text { residents }\end{array}$ & $\begin{array}{l}\text { D: Mixed } \\
\text { methods: } \\
\text { questionnaires } \\
\text { N: } 93 \\
\text { P: Residents } \\
\text { A: } 65-104 \\
\text { M: } 83.46\end{array}$ & $\begin{array}{l}\text { 'domains of quality of } \\
\text { life to accentuate the } \\
\text { positive ... [including] } \\
\text { autonomy' (p. 40). }\end{array}$ & $\begin{array}{l}\text { Experiencing } \\
\text { moments of good } \\
\text { life: uplifts. }\end{array}$ & \\
\hline $\begin{array}{l}\text { Donnelly and } \\
\text { MacEntee, } 2016\end{array}$ & Canada & 100 & $\begin{array}{l}\text { Care perceptions } \\
\text { among residents of } \\
\text { LTC facilities } \\
\text { purporting to offer } \\
\text { person-centred care }\end{array}$ & $\begin{array}{l}\text { D: Qualitative: } \\
\text { interviews } \\
\mathrm{N}: 23 \\
\text { P: Residents } \\
\text { A: } 58-97 \\
\text { M: } 83.43\end{array}$ & $\begin{array}{l}\text { 'Person-centred care } \\
\text {... enhance ... } \\
\text { autonomy' (p. 150). }\end{array}$ & $\begin{array}{l}\text { Outspoken } \\
\text { residents maintain } \\
\text { some sense of } \\
\text { autonomy. }\end{array}$ & $\begin{array}{l}\text { Staff impose } \\
\text { activities or care. }\end{array}$ \\
\hline
\end{tabular}




\begin{tabular}{|c|c|c|c|c|c|c|c|}
\hline $\begin{array}{l}\text { Dunworth and } \\
\text { Kirwan, } 2012\end{array}$ & USA & 100 & $\begin{array}{l}\text { Do nurses and social } \\
\text { workers have } \\
\text { different values? An } \\
\text { exploratory study of } \\
\text { the care for older } \\
\text { people }\end{array}$ & $\begin{array}{l}\text { D: Mixed } \\
\text { methods: } \\
\text { questionnaires } \\
\mathrm{N}: 65 \\
\mathrm{P}: \text { Staff of } \\
\text { residential care } \\
\text { facilities } \\
\mathrm{A}: 40 \geqslant 40 \text { years } \\
\text { M: - }\end{array}$ & No description. & $\begin{array}{l}\text { Care-qualified staff } \\
\text { have fewer ageist } \\
\text { assumptions. }\end{array}$ & $\begin{array}{l}\text { Non-care-qualified } \\
\text { staff give priority to } \\
\text { safety as opposed to } \\
\text { autonomy. }\end{array}$ \\
\hline Gleibs et al., 2014 & UK & 75 & $\begin{array}{l}\text { 'We get to decide': } \\
\text { the role of collective } \\
\text { engagement in } \\
\text { counteracting } \\
\text { feelings of } \\
\text { confinement and } \\
\text { lack of autonomy in } \\
\text { residential care }\end{array}$ & $\begin{array}{l}\text { D: Qualitative: } \\
\text { interviews } \\
\mathrm{N}: 10 \\
\text { P: Residents } \\
\text { A: } 76-99 \\
\text { M: - }\end{array}$ & $\begin{array}{l}\text { 'Confinement } \ldots \text { as it } \\
\text { related to ... control } \\
\text { or autonomy' (p. 268). }\end{array}$ & $\begin{array}{l}\text { Empowerment: } \\
\text { enable voice, } \\
\text { choice, control and } \\
\text { belonging through } \\
\text { group activities. } \\
\text { Helping others. } \\
\text { Social relations. }\end{array}$ & $\begin{array}{l}\text { Ageism and } \\
\text { stereotypes. Physical } \\
\text { environment: } \\
\text { needing help going } \\
\text { outdoors and to } \\
\text { other spaces. Lack of } \\
\text { control. Physical } \\
\text { mobility. }\end{array}$ \\
\hline Hall et al., 2014 & UK & 75 & $\begin{array}{l}\text { Maintaining dignity } \\
\text { for residents of care } \\
\text { homes: a qualitative } \\
\text { study of the views of } \\
\text { care home staff, } \\
\text { community nurses, } \\
\text { residents and their } \\
\text { families }\end{array}$ & $\begin{array}{l}\text { D: Qualitative: } \\
\text { interviews } \\
\mathrm{N} \text { : } 121 \\
\text { Subsets P1: } \\
\text { Staff; P2: } \\
\text { Family; P3: } \\
\text { Residents (15) } \\
\text { A: } 56-93 \text { (P3) } \\
\text { Median: } 80.5\end{array}$ & $\begin{array}{l}\text { 'Independence, } \\
\text { autonomy, choice and } \\
\text { control are related } \\
\text { concepts concerning } \\
\text { self-determination } \\
\text { (not being restrained, } \\
\text { influenced or coerced } \\
\text { by others) and not } \\
\text { relying on others for } \\
\text { aid or support' (p. 57). }\end{array}$ & $\begin{array}{l}\text { Maintain dignity } \\
\text { through: } \\
\text { independence, } \\
\text { autonomy, choice, } \\
\text { privacy, control. } \\
\text { Values of staff. } \\
\text { Communication } \\
\text { skills of staff. }\end{array}$ & $\begin{array}{l}\text { Lack of privacy. } \\
\text { Persuasion. } \\
\text { Prioritising physical } \\
\text { care and safety above } \\
\text { autonomy. Workload } \\
\text { of staff. }\end{array}$ \\
\hline
\end{tabular}

(Continued) 
Table 3. (Continued.)

\begin{tabular}{|c|c|c|c|c|c|c|c|}
\hline Authors and year & Country & $\begin{array}{l}\text { MMAT } \\
\text { quality } \\
\text { score } \\
(\%)\end{array}$ & Title of the article & $\begin{array}{l}\text { Design }(D), \\
\text { number of } \\
\text { participants }(N) \text {, } \\
\text { population }(P), \\
\text { age }(A) \text { and } \\
\text { mean age }(M)\end{array}$ & $\begin{array}{c}\text { Description of } \\
\text { autonomy (as } \\
\text { described in the } \\
\text { Introduction section) }\end{array}$ & $\begin{array}{l}\text { Facilitators (as } \\
\text { described in the } \\
\text { Results section) }\end{array}$ & $\begin{array}{c}\text { Barriers (as described } \\
\text { in the Results } \\
\text { section) }\end{array}$ \\
\hline $\begin{array}{l}\text { Hellström and } \\
\text { Sarvimäki, } 2007\end{array}$ & Sweden & 50 & $\begin{array}{l}\text { Experiences of } \\
\text { self-determination } \\
\text { by older persons } \\
\text { living in sheltered } \\
\text { housing }\end{array}$ & $\begin{array}{l}\text { D: Qualitative: } \\
\text { interviews } \\
\text { N: } 11 \\
\text { P: Residents } \\
\text { A: } 73-93 \\
\text { M: - }\end{array}$ & $\begin{array}{l}\text { '[A]utonomy was } \\
\text { conceived as } \\
\text { self-determination' } \\
\text { (p. 413). } \\
\text { 'Self-determination ... } \\
\text { is connected ... to } \\
\text { activity, mental agility, } \\
\text { survival, social } \\
\text { wellbeing, self-image } \\
\text { and health' (p. 414). }\end{array}$ & & $\begin{array}{l}\text { Disempowerment by } \\
\text { the environment, e.g. } \\
\text { lack of information } \\
\text { and shortage of staff, } \\
\text { does not strengthen } \\
\text { individual } \\
\text { self-determination, } \\
\text { participation and } \\
\text { control. Feeling } \\
\text { worthless. Being } \\
\text { immobile. }\end{array}$ \\
\hline $\begin{array}{l}\text { Hillcoat-Nallétamby, } \\
2014\end{array}$ & UK & 75 & $\begin{array}{l}\text { The meaning of } \\
\text { 'independence' for } \\
\text { older people in } \\
\text { different residential } \\
\text { settings }\end{array}$ & $\begin{array}{l}\text { D: Qualitative: } \\
\text { interviews } \\
\text { N: } 91 \\
\text { P: Residents } \\
\text { Subset NH: } \\
\text { N: } 29 \\
\text { A: } 74.5-89.5 \\
\text { M: } 82\end{array}$ & $\begin{array}{l}\text { Autonomy has the } \\
\text { dimensions: } \\
\text { delegated, decisional, } \\
\text { authentic, } \\
\text { executional, consumer } \\
\text { and direct autonomy. }\end{array}$ & $\begin{array}{l}\text { Accepting help at } \\
\text { hand. Doing things } \\
\text { alone. Having } \\
\text { friends, family and } \\
\text { financial resources. } \\
\text { Preserving physical } \\
\text { and mental } \\
\text { capacities. }\end{array}$ & \\
\hline Hwang et al., 2006 & Taiwan & 100 & $\begin{array}{l}\text { Correlates of } \\
\text { perceived autonomy } \\
\text { among elders in a } \\
\text { senior citizen home: } \\
\text { a cross-sectional } \\
\text { survey }\end{array}$ & $\begin{array}{l}\text { D: Quantitative: } \\
\text { questionnaires } \\
\text { and interviews } \\
\text { N: } 121 \\
\text { P: Residents } \\
\text { A: } 65-92 \\
\text { M: } 78\end{array}$ & $\begin{array}{l}\text { 'Autonomy ... is being } \\
\text { able to recognize } \\
\text { one's own } \\
\text { individuality, having } \\
\text { the freedom of } \\
\text { self-determination, } \\
\text { and ... the freedom to } \\
\text { act' (p. 431). }\end{array}$ & $\begin{array}{l}\text { Satisfaction of } \\
\text { social support. } \\
\text { Functional ability. } \\
\text { Life attitudes. } \\
\text { Literacy. }\end{array}$ & \\
\hline
\end{tabular}




\begin{tabular}{|c|c|c|c|c|c|c|c|}
\hline $\begin{array}{l}\text { Johnson and Bibbo, } \\
2014\end{array}$ & USA & 75 & $\begin{array}{l}\text { Relocation decisions } \\
\text { and constructing the } \\
\text { meaning of home: a } \\
\text { phenomenological } \\
\text { study of the } \\
\text { transition into a } \\
\text { nursing home }\end{array}$ & $\begin{array}{l}\text { D: Qualitative: } \\
\text { questionnaires } \\
\text { and interviews } \\
\text { N: } 18 \\
\text { P: Residents } \\
\text { A: } 68-97 \\
\text { M: - }\end{array}$ & $\begin{array}{l}\text { '[A]utonomy ... the } \\
\text { experience of having } \\
\text { freedom and/or } \\
\text { choice in daily living' } \\
\text { (p. 61). }\end{array}$ & $\begin{array}{l}\text { Self-adjustment: } \\
\text { coping with the } \\
\text { situation. Looking } \\
\text { for some autonomy } \\
\text { within the } \\
\text { institutional } \\
\text { limitations. Making } \\
\text { the decision to } \\
\text { move. A safer place } \\
\text { than the previous } \\
\text { housing. Room for } \\
\text { possessions. }\end{array}$ & $\begin{array}{l}\text { Not participating in } \\
\text { the choice to move. } \\
\text { Giving up control. } \\
\text { Lack of choice. } \\
\text { Restrictions on going } \\
\text { outside. }\end{array}$ \\
\hline Knight et al., 2010 & UK & 100 & $\begin{array}{l}\text { In home or at home? } \\
\text { How collective } \\
\text { decision making in a } \\
\text { new care facility } \\
\text { enhances social } \\
\text { interactions and } \\
\text { wellbeing among } \\
\text { older adults }\end{array}$ & $\begin{array}{l}\text { D: Quantitative: } \\
\text { questionnaires } \\
\text { and } \\
\text { observations } \\
\text { N: } 27 \\
\text { P: Residents } \\
\text { A: } 67-92 \\
\text { M: - }\end{array}$ & $\begin{array}{l}\text { Collective } \\
\text { decision-making, } \\
\text { engagement and } \\
\text { ownership. }\end{array}$ & $\begin{array}{l}\text { Enhanced by } \\
\text { participating in } \\
\text { group } \\
\text { decision-making in } \\
\text { the context of the } \\
\text { living environment. }\end{array}$ & \\
\hline Knight et al., 2011 & Australia & 100 & $\begin{array}{l}\text { Environmental } \\
\text { mastery and } \\
\text { depression in older } \\
\text { adults in residential } \\
\text { care }\end{array}$ & $\begin{array}{l}\text { D: Quantitative: } \\
\text { questionnaires } \\
\text { N: } 96 \\
\text { P: Residents } \\
\text { A: } 64-98 \\
\text { M: } 83.5\end{array}$ & $\begin{array}{l}\text { Environmental } \\
\text { mastery 'managing } \\
\text { one's environment, an } \\
\text { ability to control } \\
\text { external activities and } \\
\text { to select or develop } \\
\text { contexts suitable to } \\
\text { one's needs' (p. 875). }\end{array}$ & $\begin{array}{l}\text { Mastery leads to } \\
\text { better mental } \\
\text { health in cases of } \\
\text { illness and } \\
\text { functional } \\
\text { impairment. }\end{array}$ & \\
\hline
\end{tabular}

(Continued) 
Table 3. (Continued.)

\begin{tabular}{|c|c|c|c|c|c|c|c|}
\hline Authors and year & Country & $\begin{array}{l}\text { MMAT } \\
\text { quality } \\
\text { score } \\
(\%)\end{array}$ & Title of the article & $\begin{array}{l}\text { Design }(D), \\
\text { number of } \\
\text { participants }(N) \text {, } \\
\text { population }(P), \\
\text { age }(A) \text { and } \\
\text { mean age }(M)\end{array}$ & $\begin{array}{l}\text { Description of } \\
\text { autonomy (as } \\
\text { described in the } \\
\text { Introduction section) }\end{array}$ & $\begin{array}{l}\text { Facilitators (as } \\
\text { described in the } \\
\text { Results section) }\end{array}$ & $\begin{array}{c}\text { Barriers (as described } \\
\text { in the Results } \\
\text { section) }\end{array}$ \\
\hline Lagacé et al., 2012 & Canada & 50 & $\begin{array}{l}\text { The silent impact of } \\
\text { ageist } \\
\text { communication in } \\
\text { long term care } \\
\text { facilities: elders' } \\
\text { perspectives on } \\
\text { quality of life and } \\
\text { coping strategies }\end{array}$ & $\begin{array}{l}\text { D: Qualitative: } \\
\text { interviews } \\
\text { N: } 33 \\
\text { P: Residents } \\
\text { A: } 60-100 \\
\text { M: - }\end{array}$ & $\begin{array}{l}\text { 'Communication is an } \\
\text { essential component } \\
\text { of the caring } \\
\text { relationship, ... } \\
\text { maintain strong and } \\
\text { empowering social } \\
\text { bonds' (p. 335). }\end{array}$ & & $\begin{array}{l}\text { Ageist } \\
\text { communication leads } \\
\text { to feelings of being } \\
\text { patronised and } \\
\text { controlled: it } \\
\text { discourages } \\
\text { autonomy. Elders } \\
\text { react with avoidance } \\
\text { strategies (thus } \\
\text { reinforcing ageist } \\
\text { communication). }\end{array}$ \\
\hline Lee et al., 2013 & UK & 75 & $\begin{array}{l}\text { A narrative } \\
\text { exploration of older } \\
\text { people's transitions } \\
\text { into residential care }\end{array}$ & $\begin{array}{l}\text { D: Quantitative: } \\
\text { interviews } \\
\text { N: } 8 \\
\text { P: Residents } \\
\text { A: } 65-97 \\
\text { M: - }\end{array}$ & $\begin{array}{l}\text { Mastery over } \\
\text { environment. }\end{array}$ & $\begin{array}{l}\text { More control and } \\
\text { involvement in the } \\
\text { environment. }\end{array}$ & $\begin{array}{l}\text { Withdrawal, giving } \\
\text { up. }\end{array}$ \\
\hline $\begin{array}{l}\text { Morgan and Brazda, } \\
2013\end{array}$ & USA & 75 & $\begin{array}{l}\text { Transferring control } \\
\text { to others: process } \\
\text { and meaning of } \\
\text { older adults in } \\
\text { assisted living }\end{array}$ & $\begin{array}{l}\text { D: Qualitative: } \\
\text { interviews } \\
\text { N: } 77 \\
\text { P: Residents } \\
\text { A: } 59-99 \\
\text { M: } 85\end{array}$ & $\begin{array}{l}\text { Sense of personal } \\
\text { control in the face of } \\
\text { diminishing physical, } \\
\text { cognitive or social } \\
\text { resources. }\end{array}$ & $\begin{array}{l}\text { Transfer of control } \\
\text { to others is seen as } \\
\text { proactive and } \\
\text { positive to attain } \\
\text { goals. }\end{array}$ & $\begin{array}{l}\text { Over-helping, } \\
\text { incongruent support } \\
\text { and taking over } \\
\text { control. }\end{array}$ \\
\hline
\end{tabular}




\begin{tabular}{|c|c|c|c|c|c|c|c|}
\hline Nåden et al., 2013 & $\begin{array}{l}\text { Norway, } \\
\text { Sweden, } \\
\text { Denmark }\end{array}$ & 100 & $\begin{array}{l}\text { Aspects of indignity } \\
\text { in nursing home } \\
\text { residences as } \\
\text { experienced by } \\
\text { family caregivers }\end{array}$ & $\begin{array}{l}\text { D: Qualitative: } \\
\text { interviews } \\
\text { N: } 28 \\
\text { P: Family } \\
\text { care-givers of } \\
\text { residents (also } \\
\text { dementia) } \\
\text { A: } 47-89 \\
\text { M: - }\end{array}$ & $\begin{array}{l}\text { 'dignity ... respect for } \\
\text { personal autonomy', } \\
\text { 'central elements in } \\
\text { dignity are respect, } \\
\text { privacy and control' } \\
\text { (p. 749). }\end{array}$ & & $\begin{array}{l}\text { Being abandoned } \\
\text { and deprived of } \\
\text { dignity due to: (a) } \\
\text { feelings of not } \\
\text { belonging; (b) acts of } \\
\text { omission/offences; } \\
\text { (c) deprivation of } \\
\text { confirmations; (d) } \\
\text { physical humiliations; } \\
\text { (e) psychological } \\
\text { humiliations; (f) } \\
\text { aspects of } \\
\text { institutional life: staff } \\
\text { are not able to } \\
\text { provide good care, } \\
\text { ageist } \\
\text { communication. }\end{array}$ \\
\hline $\begin{array}{l}\text { Oosterveld-Vlug } \\
\text { et al., } 2013\end{array}$ & $\mathrm{NL}$ & 100 & $\begin{array}{l}\text { Changes in the } \\
\text { personal dignity of } \\
\text { nursing home } \\
\text { residents: a } \\
\text { longitudinal } \\
\text { qualitative interview } \\
\text { study }\end{array}$ & $\begin{array}{l}\text { D: Qualitative: } \\
\text { interviews } \\
\mathrm{N}: 30 \\
\text { P: Residents } \\
\text { A: } 49-102 \\
\text { M: - }\end{array}$ & $\begin{array}{l}\text { 'personal dignity, a } \\
\text { type of dignity that is } \\
\text { subjectively } \\
\text { experienced by an } \\
\text { individual' (p. } 1 \text { ). }\end{array}$ & $\begin{array}{l}\text { Coping mechanism } \\
\text { and professional } \\
\text { care helps to } \\
\text { maintain or regain } \\
\text { personal dignity by } \\
\text { feeling in control of } \\
\text { life. Being regarded } \\
\text { as a worthwhile } \\
\text { person by } \\
\text { themselves and } \\
\text { others. }\end{array}$ & \\
\hline $\begin{array}{l}\text { Oosterveld-Vlug } \\
\text { et al., } 2014\end{array}$ & $\mathrm{NL}$ & 100 & $\begin{array}{l}\text { Dignity and the } \\
\text { factors that } \\
\text { influence it } \\
\text { according to nursing } \\
\text { home residents: a } \\
\text { qualitative interview } \\
\text { study }\end{array}$ & $\begin{array}{l}\text { D: Qualitative: } \\
\text { interviews } \\
\text { N: } 22 \\
\text { P: Residents } \\
\text { A: } 49-97 \\
\text { M: } 77\end{array}$ & $\begin{array}{l}\text { 'Dignity ... personal, } \\
\text { subjective valuing of } \\
\text { oneself, ... valuing } \\
\text { oneself by others' } \\
\text { (p. 98). }\end{array}$ & $\begin{array}{l}\text { Preserving personal } \\
\text { dignity through } \\
\text { good professional } \\
\text { care and a } \\
\text { supportive social } \\
\text { network. }\end{array}$ & $\begin{array}{l}\text { Lack of privacy. Staff } \\
\text { not knowing the } \\
\text { resident. Leaving } \\
\text { behind loved ones. }\end{array}$ \\
\hline
\end{tabular}




\begin{tabular}{|c|c|c|c|c|c|c|c|}
\hline Authors and year & Country & $\begin{array}{l}\text { MMAT } \\
\text { quality } \\
\text { score } \\
(\%)\end{array}$ & Title of the article & $\begin{array}{l}\text { Design }(D), \\
\text { number of } \\
\text { participants }(N) \text {, } \\
\text { population }(P), \\
\text { age }(A) \text { and } \\
\text { mean age }(M)\end{array}$ & $\begin{array}{l}\text { Description of } \\
\text { autonomy (as } \\
\text { described in the } \\
\text { Introduction section) }\end{array}$ & $\begin{array}{l}\text { Facilitators (as } \\
\text { described in the } \\
\text { Results section) }\end{array}$ & $\begin{array}{c}\text { Barriers (as described } \\
\text { in the Results } \\
\text { section) }\end{array}$ \\
\hline $\begin{array}{l}\text { Palacios-Ceña et al., } \\
2013\end{array}$ & Spain & 100 & $\begin{array}{l}\text { Is the mealtime } \\
\text { experience in } \\
\text { nursing homes } \\
\text { understood? A } \\
\text { qualitative study }\end{array}$ & $\begin{array}{l}\text { D: Qualitative: } \\
\text { interviews } \\
\text { N: } 26 \\
\text { P: Residents } \\
\text { A: } \geqslant 60 \\
\text { M: } 83\end{array}$ & No description. & $\begin{array}{l}\text { Work with } \\
\text { preferences on } \\
\text { mealtimes: } \\
\text { maximise } \\
\text { autonomy, promote } \\
\text { positive social } \\
\text { experience, } \\
\text { optimise dietary } \\
\text { intake and improve } \\
\text { quality of life. }\end{array}$ & $\begin{array}{l}\text { Dining table } \\
\text { allocation by staff } \\
\text { and/or as reward or } \\
\text { punishment. }\end{array}$ \\
\hline Råholm et al., 2014 & $\begin{array}{l}\text { Norway, } \\
\text { Sweden, } \\
\text { Denmark }\end{array}$ & 100 & $\begin{array}{l}\text { Perspectives of } \\
\text { dignity of residents } \\
\text { living in nursing } \\
\text { homes: experiences } \\
\text { of family caregivers }\end{array}$ & $\begin{array}{l}\text { D: Qualitative: } \\
\text { interviews } \\
\text { N: } 28 \\
\text { P: Family } \\
\text { care-givers of } \\
\text { residents (also } \\
\text { dementia) } \\
\text { A: } 47-89 \\
\text { M: - }\end{array}$ & $\begin{array}{l}\text { ' }[1] n \text { the concept of } \\
\text { dignity: the concrete } \\
\text { level, the relational } \\
\text { level, and the } \\
\text { existential level' } \\
\text { (p. 37). }\end{array}$ & $\begin{array}{l}\text { Providing a } \\
\text { confirming culture } \\
\text { and a caring and } \\
\text { confirming } \\
\text { relationship. }\end{array}$ & $\begin{array}{l}\text { The existence of a } \\
\text { non-confirming and } \\
\text { non-caring } \\
\text { relationship. }\end{array}$ \\
\hline
\end{tabular}




\begin{tabular}{|c|c|c|c|c|c|c|c|}
\hline Sæteren et al., 2016 & $\begin{array}{l}\text { Denmark, } \\
\text { Sweden, } \\
\text { Norway }\end{array}$ & 100 & $\begin{array}{l}\text { The dialectical } \\
\text { movement between } \\
\text { deprivation and } \\
\text { preservation of a } \\
\text { person's life space }\end{array}$ & $\begin{array}{l}\text { D: Qualitative: } \\
\text { interviews } \\
\text { N: } 28 \\
\text { P: Residents } \\
\text { A: } 62-103 \\
\text { M: - }\end{array}$ & $\begin{array}{l}\text { Dignity is experienced } \\
\text { in 'maintaining } \\
\text { personal life space in } \\
\text { relation to the } \\
\text { physical environment, } \\
\text { confirming } \\
\text { interpersonal } \\
\text { relations, and a } \\
\text { feeling of inner } \\
\text { freedom and worth ... } \\
\text { despite the onset of } \\
\text { aging, disease and } \\
\text { disability' (p. 139). }\end{array}$ & $\begin{array}{l}\text { Using the inner } \\
\text { resources residents } \\
\text { have. Giving as } \\
\text { much space as } \\
\text { possible to protect } \\
\text { the residents' } \\
\text { responsibilities and } \\
\text { inner freedom. } \\
\text { Express needs and } \\
\text { desires in a negative } \\
\text { way: claim the right. }\end{array}$ & $\begin{array}{l}\text { Being dependent in } \\
\text { terms of mobility. } \\
\text { Not complaining; } \\
\text { accepting } \\
\text { withdrawal. }\end{array}$ \\
\hline $\begin{array}{l}\text { Sikorska-Simmons, } \\
2006\end{array}$ & USA & 75 & $\begin{array}{l}\text { The effects of } \\
\text { organizational } \\
\text { policies on resident } \\
\text { perceptions of } \\
\text { autonomy in } \\
\text { assisted living }\end{array}$ & $\begin{array}{l}\text { D: Quantitative: } \\
\text { questionnaires } \\
\text { N: } 412 \\
\text { P: Residents } \\
\text { A: } 59-101 \\
\text { M: } 83\end{array}$ & $\begin{array}{l}\text { Decisional autonomy: } \\
\text { 'perceptions of } \\
\text { influence and } \\
\text { independence' (p. 62). }\end{array}$ & $\begin{array}{l}\text { Higher levels of } \\
\text { choice-enhancing } \\
\text { policies. }\end{array}$ & \\
\hline $\begin{array}{l}\text { Walent and } \\
\text { Kayser-Jones, } 2008\end{array}$ & USA & 75 & $\begin{array}{l}\text { Having a voice and } \\
\text { being heard: nursing } \\
\text { home residents and } \\
\text { in-house advocacy }\end{array}$ & $\begin{array}{l}\text { D: Qualitative: } \\
\text { interviews and } \\
\text { document } \\
\text { review } \\
\text { N: } 17 \\
\text { P: Residents } \\
\text { A: } 66-94 \\
\text { M: - }\end{array}$ & $\begin{array}{l}\text { 'Self-advocacy ... } \\
\text { ability to voice one's } \\
\text { own needs and } \\
\text { concerns and to } \\
\text { persist until they were } \\
\text { heard and met' } \\
\text { (p. 39). }\end{array}$ & $\begin{array}{l}\text { Formal advocacy: } \\
\text { the organisation } \\
\text { promotes the } \\
\text { residents' voice by } \\
\text { pairing employees } \\
\text { with residents. } \\
\text { Residents who are } \\
\text { advocates for other } \\
\text { residents. }\end{array}$ & $\begin{array}{l}\text { Personal, } \\
\text { organisational, social } \\
\text { and cultural barriers. }\end{array}$ \\
\hline $\begin{array}{l}\text { Walker and } \\
\text { Paliadelis, } 2016\end{array}$ & Australia & 100 & $\begin{array}{l}\text { Older peoples' } \\
\text { experiences of living } \\
\text { in a residential aged } \\
\text { care facility in } \\
\text { Australia }\end{array}$ & $\begin{array}{l}\text { D: Qualitative: } \\
\text { interviews } \\
\text { N: } 18 \\
\text { P: Residents } \\
\text { A: } 77-96 \\
\text { M: - }\end{array}$ & $\begin{array}{l}\text { 'losing control over } \\
\text { making life choices' } \\
\text { (p. e7). }\end{array}$ & $\begin{array}{l}\text { Meaningful relations } \\
\text { with staff, family } \\
\text { and friends. } \\
\text { Acceptance. Making } \\
\text { the best. }\end{array}$ & $\begin{array}{l}\text { Trading } \\
\text { independency for } \\
\text { safety and assistance } \\
\text { in NH. Exclusion from } \\
\text { formal } \\
\text { decision-making. }\end{array}$ \\
\hline
\end{tabular}

Notes: 1. Given in the Results section. MMAT: Mixed Methods Appraisal Tool. NL: The Netherlands. USA: United States of America. NH: nursing home. ADL: activities of daily living. LTC: long-term care. 
100 per cent. Generally speaking, the methodological quality of the articles is appropriate: the mean quality score is 82.9 per cent and 19 articles score 100 per cent. Column 5 shows us the designs ('D'). Qualitative designs (23 articles, $65.7 \%$ ) were used most frequently, followed by quantitative (nine articles, $25.7 \%$ ) and mixed-methods designs (three articles, 8.6\%). Interviewing (22) is the method most used. In three articles, these interviews are combined with questionnaires and one of the interview studies is combined with a document review. There are ten questionnaire studies, of which two combined the questionnaire with observations. The other methods used in the articles are observation (two) and action research (one). Seven articles evaluated the effect of interventions on autonomy. In 32 of the 35 articles (see column 5, 'P'), the perspective of the resident was explored.

\section{Description of autonomy in the included articles}

For a better understanding of the facilitators and barriers to autonomy, the authors first aim to underpin the concept of autonomy for residents with physical impairments. The word autonomy is used in 16 articles (see column 6, description of autonomy, in Table 3). The polarity of decisional and executional autonomy (Collopy, 1988) was mentioned in four articles (Hwang et al., 2006; Sikorska-Simmons, 2006; Hellström and Sarvimäki, 2007; Hillcoat-Nallétamby, 2014). Most of the other included articles only used one element of the polarity, the decisional aspect.

Autonomy, self-determination and dignity seem to be linked. Various relationships between these concepts were described in the included articles, as causes, intermediate factors or outcomes of one another. For example, dignity as a cause for autonomy (Nåden et al., 2013). Also, an opposite perspective is mentioned: autonomy, amongst other aspects, leads to dignity (Hall et al., 2014). Dignity as a result of choice and autonomy is also described (Oosterveld-Vlug et al., 2014). Three articles use the motivational theory of Ryan and Deci (2000): in this theory, autonomy leads to self-determination (Custers et al., 2010, 2011,2012). Self-determination is also seen as a sub-category of autonomy (Hellström and Sarvimäki, 2007).

Based on the elements from column 6 of Table 3 (description of autonomy), a description of autonomy was formulated in such a way that it best matches the population in this review: older residents with physical impairments in RCFs. In the current article, autonomy is described as a capacity to influence the environment (Knight et al., 2011; Lee et al., 2013; Sæteren et al., 2016) and make decisions (Bolmsjö et al., 2006; Hwang et al., 2006; Sikorska-Simmons, 2006; Hellström and Sarvimäki, 2007; Walent and Kayser-Jones, 2008; Andresen et al., 2009; Cooney et al., 2009; Anderberg and Berglund, 2010; Custers et al., 2010, 2011, 2012; Knight et al., 2010; Dunworth and Kirwan, 2012; Morgan and Brazda, 2013; Nåden et al., 2013; Gleibs et al., 2014; Hall et al., 2014; Hillcoat-Nallétamby, 2014; Johnson and Bibbo, 2014), irrespective of having executional autonomy (Hillcoat-Nallétamby, 2014), to live the kind of life someone desires to live (Bolmsjö et al., 2006; Walent and Kayser-Jones, 2008; Andresen et al., 2009; Custers et al., 2010, 2011, 2012; Knight et al., 2010; Johnson and Bibbo, 2014; Sæteren et al., 2016; Walker and Paliadelis, 2016) in the face of diminishing social, physical and/or cognitive resources and dependency (Morgan and Brazda, 
2013; Sæteren et al., 2016), and it develops in relationships (Bolmsjö et al., 2006; Knight et al., 2010; Abma et al., 2012; Baur and Abma, 2012; Lagacé et al., 2012; Oosterveld-Vlug et al., 2013, 2014; Palacios-Ceña et al., 2013; Sæteren et al., 2016; Walker and Paliadelis, 2016).

\section{Facilitators and barriers to autonomy of older adults with physical impairments in RCFs}

The results of the literature review are organised into four themes of which characteristics of residents is the first theme. This theme is based on the included literature that provided rich information on the older adults themselves. The other themes are derived from the PCC framework: prerequisites of professional caregivers in RCFs, processes in the relationship between residents and professional care-givers, and the care environment (McCormack and McCance, 2017). Often the results reveal an ambiguity: aspects can either be facilitators or barriers. These will be elaborated on below, starting with the resident characteristics.

\section{Characteristics of residents: facilitators}

First, psycho-social characteristics of residents were identified (Table 4). Visits from family and friends help older adults to experience a sense of continuity of the life they lived before moving into the RCF (Hillcoat-Nallétamby, 2014). As a consequence of these visits, the valued roles they used to have for family and friends can be maintained. This offers a sense of belonging and autonomy (Hwang et al., 2006; Chao et al., 2008; Walent and Kayser-Jones, 2008; Cooney et al., 2009; Oosterveld-Vlug et al., 2014). If older adults have financial resources, possibilities are created to make decisions on spending money and having choice and control in their lives in RCF (Chao et al., 2008; Gleibs et al., 2014; Hillcoat-Nallétamby, 2014). The presence of meaningful activities can give control and social engagement. Through these activities, older adults can help each other and, as a result, have useful recognised roles (Danhauer et al., 2006; Walent and Kayser-Jones, 2008; Custers et al., 2012; Gleibs et al., 2014; Hillcoat-Nallétamby, 2014; Råholm et al., 2014).

Also, diverse intrapersonal characteristics are distinguished. Coping skills, which older adults developed earlier in their life history, lead to more control over the situation and autonomy (Hwang et al., 2006; Curtiss et al., 2007; Cooney et al., 2009; Anderberg and Berglund, 2010; Custers et al., 2010; Brandburg et al., 2013; Oosterveld-Vlug et al., 2013, 2014; Donnelly and MacEntee, 2016; Walker and Paliadelis, 2016). Relations with staff are important for exercising autonomy. In these relationships, older adults' need to be regarded as worthwhile persons can be fulfilled. Especially when residents lack family and friends who can act as advocates, relations with staff become more important (Chao et al., 2008; Walent and Kayser-Jones, 2008; Oosterveld-Vlug et al., 2013, 2014; Gleibs et al., 2014; Hillcoat-Nallétamby, 2014). The possibility of deciding themselves about moving into the facility seems to have a positive impact on the feeling of autonomy and control (Chao et al., 2008; Lee et al., 2013; Morgan and Brazda, 2013; Johnson and Bibbo, 2014). 
Table 4. Characteristics of residents

\begin{tabular}{|c|c|}
\hline Facilitators & Barriers \\
\hline \multicolumn{2}{|l|}{ Psycho-social characteristics: } \\
\hline $\begin{array}{l}\text { - Having financial resources } \\
\text { - Helping family and friends } \\
\text { - Relations with staff } \\
\text { - Group activities } \\
\text { - Social engagement } \\
\text { - Helping each other }\end{array}$ & $\begin{array}{l}\text { - Absence of family and friends } \\
\text { - Over-helping by others } \\
\text { - Incongruent support } \\
\text { - Leaving behind a husband or wife } \\
\text { - Not complaining } \\
\text { - Interpreting that staff are too busy }\end{array}$ \\
\hline \multicolumn{2}{|l|}{ Intrapersonal characteristics: } \\
\hline $\begin{array}{l}\text { - Having a sense of meaning } \\
\text { - Continuity of identity } \\
\text { - Awareness } \\
\text { - Coping abilities } \\
\text { - Learning abilities } \\
\text { - Uplifts } \\
\text { - Taking responsibility } \\
\text { - Educational level } \\
\text { - Decision-making on moving } \\
\text { - Information seeking } \\
\text { - Optimism and hope } \\
\text { - Mastering life in the institution and the } \\
\text { wisdom to accept } \\
\text { - Positive attitude } \\
\text { - Feeling of being in control } \\
\text { - Cognitive functions }\end{array}$ & $\begin{array}{l}\text { - Being unable to make decisions on } \\
\text { moving } \\
\text { - Sense of ineffectiveness } \\
\text { - Acceptance of rules instead of } \\
\text { questioning them }\end{array}$ \\
\hline \multicolumn{2}{|l|}{ Physical functioning: } \\
\hline - Residential care facility offers protection & $\begin{array}{l}\text { - Diminished ability to communicate } \\
\text { - Being dependent }\end{array}$ \\
\hline
\end{tabular}

The last characteristic of the residents is the level of physical functioning. A higher level results in more control and choice in activities. Also, there is a higher use of living and other spaces in the RCF. In addition, there are more possibilities for going out (Hwang et al., 2006; Chao et al., 2008; Oosterveld-Vlug et al., 2013; Hillcoat-Nallétamby, 2014).

\section{Characteristics of residents: barriers}

As said before, the aspects reveal an ambiguity, they can either be facilitator or barrier. The barriers are now given for the same aspects as above.

Psycho-social characteristics were identified, such as the absence of family and friends. In addition, being over-helped by others or receiving incongruent support are barriers to autonomy (Walent and Kayser-Jones, 2008; Morgan and Brazda, 2013; Oosterveld-Vlug et al., 2014). If older adults do not have family and friends, they have to rely on staff or other residents for attention and help. Often older 
adults hesitate to state their wishes and needs. They suppose that staff are too busy. Sometimes older adults assume that complaining or asking for help will have a negative effect on the care they receive (Bolmsjö et al., 2006; Hellström and Sarvimäki, 2007; Walent and Kayser-Jones, 2008; Cooney et al., 2009; Lagacé et al., 2012; Morgan and Brazda, 2013; Donnelly and MacEntee, 2016).

Barriers in the intrapersonal characteristics, such as being unable to participate in the decision-making process of moving into the RCF, affect autonomy negatively (Chao et al., 2008; Cooney et al., 2009; Johnson and Bibbo, 2014; Lee et al., 2013). Furthermore, shared decision-making is not taken for granted, because rules and time schedules are often accepted by older adults (Hellström and Sarvimäki, 2007; Chao et al., 2008; Walent and Kayser-Jones, 2008; Lee et al., 2013; Morgan and Brazda, 2013; Johnson and Bibbo, 2014; Donnelly and MacEntee, 2016; Sæteren et al., 2016; Walker and Paliadelis, 2016).

In physical functioning, as the last characteristic of residents, barriers are also found. Immobility and a diminished ability to communicate might act as barriers. A lack of energy can interfere with residents being able to live the lives they want to live (Hellström and Sarvimäki, 2007; Walent and Kayser-Jones, 2008; Oosterveld-Vlug et al., 2013, 2014; Gleibs et al., 2014).

\section{Prerequisites of professional care-givers in RCFs: facilitators}

The second theme used to organise the results is the prerequisites of professional care-givers in RCFs (Table 5). The awareness of beliefs and values is established as prerequisite. Staff who are able to provide good professional care and are able to build high-quality relationships with residents help to preserve autonomy. So do staff who are able to treat residents with respect. The ability to take care of the physical appearance of residents also enhances autonomy (Custers et al., 2011; Oosterveld-Vlug et al., 2014; Råholm et al., 2014).

More highly educated nurses, and nurses in higher positions, seem to be more capable of supporting autonomy. They are more reflective in their attitude and have fewer ageist assumptions (Dunworth and Kirwan, 2012).

Also, ethical competence and creativity of the staff are seen as facilitating autonomy (Bolmsjö et al., 2006; Hall et al., 2014).

\section{Prerequisites of professional care-givers in RCFs: barriers}

Barriers are also seen in the prerequisites. Dissatisfaction with the job and lack of ethical competence are barriers to autonomy. Negative beliefs and values such as ageist assumptions in staff, expressed in ageist communication and adverse relationships, are also barriers to autonomy (Bolmsjö et al., 2006; Dunworth and Kirwan, 2012; Gleibs et al., 2014; Hall et al., 2014). An example of unethical behaviour in staff is seen when tables in the dining rooms are allocated as a punishment or reward for certain behaviours of older adults, thus leaving residents no choice of dinner companions (Palacios-Ceña et al., 2013). Another threat to autonomy is undignified care, like forced-feeding situations (Nåden et al., 2013).

Often encounters between staff and residents are scarce and show a lack of reciprocity. The last aspect in this theme is that staff seem unable to identify the 
Table 5. Prerequisites of professional care-givers in residential care facilities

\begin{tabular}{|c|c|}
\hline Facilitators & Barriers \\
\hline \multicolumn{2}{|l|}{ Beliefs and values: } \\
\hline $\begin{array}{l}\text { - The ward covers a set of positive values which is } \\
\text { reflected in the actions of staff }\end{array}$ & $\begin{array}{l}\text { - Ageist assumptions } \\
\text { - Stereotyping and stigmatising } \\
\text { - Absence of underlying values }\end{array}$ \\
\hline \multicolumn{2}{|l|}{ Relationship: } \\
\hline - High-quality, caring relationships & $\begin{array}{l}\text { - Coercing relationships: } \\
\text { punishing, rewarding }\end{array}$ \\
\hline \multicolumn{2}{|l|}{ Commitment to the job: } \\
\hline - Higher educational level or job function & - No satisfaction with the job \\
\hline \multicolumn{2}{|l|}{ Ethical competence: } \\
\hline $\begin{array}{l}\text { - Reflection } \\
\text { - Creativity }\end{array}$ & $\begin{array}{l}\text { - Talking about choice but not } \\
\text { acting upon it } \\
\text { - Not reacting to individuals' } \\
\text { needs }\end{array}$ \\
\hline \multicolumn{2}{|l|}{ Communication skills: } \\
\hline - Confirming communication & - Ageist communication \\
\hline
\end{tabular}

underlying messages in the communication. This can lead to an unfulfilled desire for autonomy (Bolmsjö et al., 2006).

\section{Processes in the relationship between residents and professional care-givers: facilitators}

Communication is the first aspect that is distinguished in the processes between residents and care-givers (Table 6). Staff who have a good relationship with the older adults contribute to their need fulfilment. So do respectful communication and care for their physical appearance (Custers et al., 2010).

Relations between residents and staff can reveal how diverse adaptive strategies are applied by older adults to have a life of their own in the RCF. Knowing and working with these individual strategies facilitates autonomy and assists older adults in dealing with problems (Andresen et al., 2009; Brandburg et al., 2013). 
Table 6. Processes in the relation between residents and professional care-givers

\begin{tabular}{|c|c|}
\hline Facilitators & Barriers \\
\hline \multicolumn{2}{|l|}{ Communication: } \\
\hline - Shared decision-making & $\begin{array}{l}\text { - Talking about persons } \\
\text { - Persuasion }\end{array}$ \\
\hline \multicolumn{2}{|l|}{ Relations: } \\
\hline $\begin{array}{l}\text { - Friendly, trustful relationships } \\
\text { - Respecting and knowing the person and her or his past } \\
\text { - Reinforcing, empowering } \\
\text { - Reciprocity }\end{array}$ & $\begin{array}{l}\text { - No information and choice } \\
\text { - Conflicts }\end{array}$ \\
\hline \multicolumn{2}{|l|}{ Care-giving: } \\
\hline $\begin{array}{l}\text { - Giving space } \\
\text { - Individual tailored programmes }\end{array}$ & $\begin{array}{l}\text { - Prioritising physical care } \\
\text { - Having to wait, ignoring } \\
\text { - Physical humiliations }\end{array}$ \\
\hline
\end{tabular}

Staff can find out what autonomy means for older adults by listening to life stories. These stories reflect the values of older adults in life, their personal identity and relations (Abma et al., 2012). With an empowering strategy, involvement in care and shared goals can be realised and ownership is enhanced (Baur and Abma, 2012; Sæteren et al., 2016).

\section{Processes in the relationship between residents and professional care-givers: barriers}

The lack of constructive communication can act as a barrier to autonomy. For example, when staff use routines, or impose activities of care or let older adults wait for help (Nåden et al., 2013; Palacios-Ceña et al., 2013; Oosterveld-Vlug et al., 2014; Donnelly and MacEntee, 2016). The possibility of participating in decision-making can be hindered by a lack of information and choice (Hellström and Sarvimäki, 2007; Walent and Kayser-Jones, 2008). Furthermore, conflicts with staff might discourage older adults from expressing their wants and needs (Walent and Kayser-Jones, 2008; Lagacé et al., 2012; Hall et al., 2014).

\section{Care environment: facilitators}

The last theme to organise the results is the care environment (Table 7). RCFs that have high levels of choice-enhancing policies and have adequate staff seem to increase the residents' autonomy. Also, financial resources and a conforming 
Table 7. Care environment

\begin{tabular}{ll}
\hline Facilitators & \multicolumn{1}{c}{ Barriers } \\
\hline Choice and control: & - Schedules and regulations \\
\hline $\begin{array}{l}\text { - Formal involvement in } \\
\text { decision-making }\end{array}$ & $\begin{array}{l}\text { - Ock of choice } \\
\text { professional }\end{array}$ \\
\hline Supportive systems & \\
\hline Staffing: & - Inadequate staffing \\
\hline - Skilled personnel & Deployment of temporary personnel \\
\hline Ethnical and cultural congruity & \\
\hline Background of management & \\
\hline Physical and financial environment: & \\
\hline Shared and private spaces & - Lack of resources \\
\hline
\end{tabular}

physical outline seem to act as facilitators (Sikorska-Simmons, 2006; Walent and Kayser-Jones, 2008; Knight et al., 2010). For example, the management can support the participation of older adults in organisational choices, such as selection of menu, gardening and social activities. This enhances the sense of mastery (Knight et al., 2011; Baur and Abma, 2012; Lee et al., 2013). Another example is the employment of skilled and permanent staff who share the same language, which facilitates autonomy (Walent and Kayser-Jones, 2008; Cooney et al., 2009; Custers et al., 2011; Walker and Paliadelis, 2016; Dunworth and Kirwan, 2012). A combination of appropriate shared and private spaces for older adults enhances choice, feelings of safety and participation (Chao et al., 2008; Hall et al., 2014; Hillcoat-Nallétamby, 2014; Johnson and Bibbo, 2014).

\section{Care environment: barriers}

A lack of choice and control in daily life, such as the use of schedules, is found as a barrier. These schedules force older adults to fit their lives into routines, which might undermine autonomy. Also, routines for activities such as morning procedures, meals, washing, going to the toilet and bedtimes can act as barriers to autonomy (Curtiss et al., 2007; Andresen et al., 2009; Hall et al., 2014; Johnson and Bibbo, 2014).

Understaffing and employment of temporary employees can be barriers to autonomy. There is no time to get acquainted, to build relationships and to get to know the preferences of residents (Walent and Kayser-Jones, 2008; Hall et al., 2014; Oosterveld-Vlug et al., 2014). 
Shortages in resources due to directives and political decisions is one of the causes of understaffing. This affects autonomy because there are fewer staff to respond to older adults' needs (Hellström and Sarvimäki, 2007; Gleibs et al., 2014). The physical outline of the building and decoration of the rooms influence the experience of feeling at home. RCFs that appear like a hospital have a nonconfirming atmosphere (Cooney et al., 2009; Lagacé et al., 2012; Nåden et al., 2013; Gleibs et al., 2014; Oosterveld-Vlug et al., 2014).

\section{Discussion}

The current literature review was executed to gain more insight into facilitators and barriers to autonomy of residents with physical impairments living in RCFs. Based on the literature search and the subsequent synthesis of the data of the included articles, the facilitators and barriers to autonomy were identified and organised. Three themes were based on the framework of PCP (McCormack and McCance, 2017). Particular aspects in the care environment act as barriers to autonomy. Relationships between staff and residents can either facilitate or inhibit autonomy, depending on the prerequisites of the care-givers and characteristics, e.g. coping skills, of the residents.

Although the framework includes elements of PCP, the care recipient heror himself is not present in the model. In the current review, characteristics of residents that influence autonomy were determined. The theme 'characteristics of residents' is added to arrange the results of the older adults. The majority of the articles investigated this perspective, so a large set of attributes of residents that influence autonomy were distinguished.

Facilitators and barriers to autonomy can be allocated to elements of the PCP framework. The macro context, which contains aspects such as health policies and strategic frameworks, is not investigated in the included articles. The PCP framework seems to encompass all the distinguished influencing aspects for autonomy in the included articles. The culture change to more person-centred care can enhance autonomy. Realising a culture change in RCFs, however, is difficult with so many challenges to deal with (Donnelly and MacEntee, 2016).

Based on the descriptions of the included articles, a description of autonomy was formulated. The authors established this description because it compiles the core elements of autonomy for older adults with physical impairments living in RCFs, as used in the included articles. Autonomy is described as a capacity to influence the environment (Knight et al., 2011; Lee et al., 2013; Sæteren et al., 2016) and make decisions (Bolmsjö et al., 2006; Hwang et al., 2006; Sikorska-Simmons, 2006; Hellström and Sarvimäki, 2007; Walent and Kayser-Jones, 2008; Andresen et al., 2009; Cooney et al., 2009; Anderberg and Berglund, 2010; Custers et al., 2010, 2011, 2012; Knight et al., 2010; Dunworth and Kirwan, 2012; Morgan and Brazda, 2013; Nåden et al., 2013; Gleibs et al., 2014; Hall et al., 2014; Hillcoat-Nallétamby, 2014; Johnson and Bibbo, 2014), irrespective of having executional autonomy (Hillcoat-Nallétamby, 2014), to live the kind of life someone desires to live (Bolmsjö et al., 2006; Walent and Kayser-Jones, 2008; Andresen et al., 2009; Custers et al., 2010, 2011, 2012; Knight et al., 2010; Johnson and Bibbo, 2014; Sæteren et al., 2016; Walker and Paliadelis, 2016) in the face of 
diminishing social, physical and/or cognitive resources and dependency (Morgan and Brazda, 2013; Sæteren et al., 2016), and it develops in relationships (Bolmsjö et al., 2006; Knight et al., 2010; Abma et al., 2012; Baur and Abma, 2012; Lagacé et al., 2012; Oosterveld-Vlug et al., 2013, 2014; Palacios-Ceña et al., 2013; Sæteren et al., 2016; Walker and Paliadelis, 2016).

Based on the included articles, the description focuses on decisional and relational autonomy. This might be explained because the literature search was performed for physically impaired older adults living in RCFs. These residents are generally able to make choices, but physical impairments can obstruct the execution of the decisions taken. They often need practical help from others to carry out their decisions. Also, the relational aspect was prominent in the included articles, which can be related to the fact that living in an RCF means living with other residents and staff, and thus in relation to others.

The aspect of forced autonomy, using force to make decisions and act upon them independently, was not present in the included articles. However, paternalism was present: making choices for persons who are able to make decisions on their own.

Nonetheless, we found barriers to autonomy related to force, for example the forced use of services, such as eating, following regulations, transfer to the residential care, and transfer of tasks and responsibilities. Autonomy, in the included articles, is often hindered by care-givers and institutions, and is not forced upon residents (Hwang et al., 2006; Hellström and Sarvimäki, 2007; Knight et al., 2011; Dunworth and Kirwan, 2012; Lagacé et al., 2012; Oosterveld-Vlug et al., 2014).

\section{Strengths}

In this review, results from articles that focus on dignity, self-determination and autonomy are aggregated. The different positions in the relationships between the three concepts and their intertwined use in the included articles made it rewarding to merge all facilitators and barriers. As a result of the merging, the review offers a comprehensive overview of factors that influence autonomy of residents with physical impairments living in RCFs.

The execution of the systematic review by four of the five authors was established first independently and later through meetings to achieve a uniform procedure at the start of each stage of the selection, quality assessment and data extraction. The first author assessed all articles. Three of the co-authors reviewed a selection of the articles. At each stage of the selection process, consensus was reached about inclusion or exclusion of articles by means of bilateral discussions.

\section{Limitations}

A limitation in organising the results according to the PCP framework is that some of the included articles lack specific information, so the allocation of a facilitator or barrier can be difficult. For example, the framework makes a distinction between being prepared for the job (prerequisites) and delivering care (person-centred processes). However, there is not enough information in the included articles about preparation for the job or educational background. The consequence is that it is difficult to allocate results such as communication to either prerequisites 
(communication skills) or care processes (communication). The same can be said for building relationships (prerequisite) and relations (care processes). In that case, barriers and facilitators were allocated to both themes, so a repetition is seen.

In this study, the authors aimed to include residents with physical impairments. However, it cannot be certain that persons with dementia were totally excluded because of the lack of precise information about the assessment of mental status. The content of the articles, however, gives confidence that the research is not done on persons with moderate or severe dementia.

The same can be said for the inclusion of persons with an average age of 65 years. The authors screened the articles thoroughly to exclude studies on residents under 65 years. However, if some individuals under this age participated in the studies, the authors calculated the mean age. The mean in all these articles was 77 years or more. This mean of 77 was used as a rationale to include the article as describing residents above 65 years. For three included articles, the authors were not able to calculate a mean age because the individual ages of the participants were not given. However, an age range of 49-102 (Oosterveld-Vlug et al., 2013), 62-103, (Sæteren et al., 2016) and 60-100 (Lagacé et al., 2012) was provided for the participants of their studies. The subject of the articles gives us the assurance that the group had age-related impairments.

\section{Implications for practice and science}

The current review leads to a better understanding of autonomy-enhancing elements for residents with physical impairments in RCFs. Autonomy is a broad, complex, multifaceted and relational concept that can be influenced by many factors in various ways. The results have implications for practice for both residents and care-givers, because they offer possibilities to preserve and enhance autonomy. The knowledge of facilitators and barriers established in this review can be used in the education of current and future nurses or other care personnel to make them aware of how to enhance autonomy. Based on the results in all four themes, RCFs can systematically develop autonomy-enhancing practices.

Scientifically, this study creates new knowledge and provides an actual overview on autonomy for older adults with physical impairments in RCFs and how to support autonomy. The results accentuate the influence of multiple aspects to achieve autonomy in RCFs.

More empirical research should be done on autonomy in practice. What significance does autonomy have for residents and staff and when is autonomy (not) enhanced or perhaps forced? Do we recognise (parts of) the description of autonomy in daily care practice? Because autonomy is a complex, relational and dynamic concept, it can best be investigated through observational methods that examine the perspectives of residents and care-givers. Shadowing is a method that can be used in an environment where autonomy is manifested, e.g. in RCFs (van der Meide et al., 2015). Research can give insight into how factors established in this review interrelate and how they are expressed in the care process. It is advisable to investigate dimensions of the concept of autonomy other than executional and decisional autonomy which dominate in the results of this systematic review. It is possible that important aspects of autonomy - e.g. the relational aspect of autonomy - are 
getting less attention or can be overlooked if further research restricts itself to this polarity. More attention should also be paid to the facilitators and barriers in the macro context. RCFs are strongly dependent on government health policies and funding to achieve autonomy-enhancing practices.

Furthermore, the knowledge can be used in participatory transformational action research. Action groups with different stakeholders in RCFs can experiment with actions to strengthen autonomy. In this way, the perspectives of residents, care-givers and organisations can be studied in relation to each other. Supportive practices for autonomy can be identified and examined by means of this bottom-up development.

Author contributions. JvL performed the literature search and selection, assessed the quality of the selected articles, extracted data and constructed the tables. KL, IdR and BJ selected the articles, assessed the quality of the selected articles and extracted data. All the authors, including MJ, interpreted the findings and were involved in the drafting and revisions of the manuscript. They approved the publication of the article.

Financial support. This work was supported by De Wever, a care organisation in Tilburg. De Wever had no role in the research.

Conflict of interest. The authors declare no conflicts of interest.

Ethical standards. Not applicable for a literature review.

\section{References}

Abma T, Bruijn A, Kardol T, Schols J and Widdershoven G (2012) Responsibilities in elderly care: $\mathrm{Mr}$ Powell's narrative of duty and relations. Bioethics 26, 22-31.

Anderberg P and Berglund A (2010) Elderly persons' experiences of striving to receive care on their own terms in nursing homes. International Journal of Nursing Practice 16, 64-68.

Andresen M, Runge U, Hoff M and Puggaard L (2009) Perceived autonomy and activity choices among physically disabled older people in nursing home settings: a randomized trial. Journal of Aging \& Health 21, 1133-1158.

Baur V and Abma T (2012) 'The Taste Buddies': participation and empowerment in a residential home for older people. Ageing \& Society 32, 1055-1078.

Bjornsdottir K (2009) The ethics and politics of home care. International Journal of Nursing Studies 46, 732-739.

Bolmsjö IA, Sandman L and Andersson E (2006) Everyday ethics in the care of elderly people. Nursing Ethics 13, 249-263.

Brandburg GL, Symes L, Mastel-Smith B, Hersch G and Walsh T (2013) Resident strategies for making a life in a nursing home: a qualitative study. Journal of Advanced Nursing 69, 862-874.

Cartier C (2003) From home to hospital and back again: economic restructuring, end of life, and the gendered problems of place-switching health services. Social Science and Medicine 56, 2289-2301.

Chao S, Lan Y, Tso H, Chung C, Neim Y and Clark MJ (2008) Predictors of psychosocial adaptation among elderly residents in long-term care settings. Journal of Nursing Research (Taiwan Nurses Association) 16, 149-158.

Collopy BJ (1988) Autonomy in long term care: some crucial distinctions. The Gerontologist 28, supplement, $10-17$.

Cooney A, Murphy K and O'Shea E (2009) Resident perspectives of the determinants of quality of life in residential care in Ireland. Journal of Advanced Nursing 65, 1029-1038.

Curtiss K, Hayslip B and Dolan DC (2007) Motivational style, length of residence, voluntariness, and gender as influences on adjustment to long term care: a pilot study. Journal of Human Behavior in the Social Environment 15, 13-34. 
Custers AFJ, Kuin Y, Riksen-Walraven M and Westerhof GJ (2011) Need support and wellbeing during morning care activities: an observational study on resident-staff interaction in nursing homes. Ageing \& Society 31, 1425-1442.

Custers AFJ, Westerhof GJ, Kuin Y, Gerritsen DL and Riksen-Walraven JM (2012) Relatedness, autonomy, and competence in the caring relationship: the perspective of nursing home residents. Journal of Aging Studies 26, 319-326.

Custers AFJ, Westerhof GJ, Kuin Y and Riksen-Walraven M (2010) Need fulfillment in caring relationships: its relation with well-being of residents in somatic nursing homes. Aging \& Mental Health 14, 731-739.

Danhauer SC, Sorocco KH and Andrykowski MA (2006) Accentuating the positive: recent 'uplifts' reported by nursing home residents. Clinical Gerontologist 29, 39-58.

Donnelly L and MacEntee MI (2016) Care perceptions among residents of LTC facilities purporting to offer person-centred care. Canadian Journal on Aging 35, 149-160.

Dunworth M and Kirwan P (2012) Do nurses and social workers have different values? An exploratory study of the care for older people. Journal of Interprofessional Care 26, 226-231.

Gleibs IH, Sonnenberg SJ and Haslam C (2014) 'We get to decide': the role of collective engagement in counteracting feelings of confinement and lack of autonomy in residential care. Activities, Adaptation \& Aging 38, 259-280.

Hall S, Dodd RH and Higginson IJ (2014) Maintaining dignity for residents of care homes: a qualitative study of the views of care home staff, community nurses, residents and their families. Geriatric Nursing 35, 55-60.

Hellström UW and Sarvimäki A (2007) Experiences of self-determination by older persons living in sheltered housing. Nursing Ethics 14, 413-424.

Hillcoat-Nallétamby S (2014) The meaning of 'independence' for older people in different residential settings. Journals of Gerontology: Psychological Sciences and Social Sciences 69B, 419-430.

Hwang H-L, Lin H-S, Tung Y-L and Wu H-C (2006) Correlates of perceived autonomy among elders in a senior citizen home: a cross-sectional survey. International Journal of Nursing Studies 43, 429-437.

Jacobs G (2019) Patient autonomy in home care: nurses' relational practices of responsibility. Nursing Ethics 26, 1638-1653.

Johnson RA and Bibbo J (2014) Relocation decisions and constructing the meaning of home: a phenomenological study of the transition into a nursing home. Journal of Aging Studies 30, 56-63.

Knight C, Haslam SA and Haslam C (2010) In home or at home? How collective decision making in a new care facility enhances social interaction and wellbeing amongst older adults. Ageing \& Society $\mathbf{3 0}$, 1393-1418.

Knight T, Davison TE, McCabe MP and Mellor D (2011) Environmental mastery and depression in older adults in residential care. Ageing \& Society 31, 870-884.

Lagacé M, Tanguay A, Lavallée M-L, Laplante J and Robichaud S (2012) The silent impact of ageist communication in long term care facilities: elders' perspectives on quality of life and coping strategies. Journal of Aging Studies 26, 335-342.

Lee VS, Simpson J and Froggatt K (2013) A narrative exploration of older people's transitions into residential care. Aging Mental Health 17, 48-56.

McCormack B (2001) Autonomy and the relationship between nurses and older people. Ageing \& Society 21, 417-446.

McCormack B and McCance T (2017) Person-centred Practice in Nursing and Health Care: Theory and Practice. Chichester, UK: John Wiley and Sons.

Morgan LA and Brazda MA (2013) Transferring control to others: process and meaning for older adults in assisted living. Journal of Applied Gerontology 32, 651-668.

Nåden D, Rehnsfeldt A, Råholm M-B, Lindwall L, Caspari S, Aasgaard T, Slettebø Å, Sæteren B, Høy B, Lillestø B, Heggestad AKT and Lohne V (2013) Aspects of indignity in nursing home residences as experienced by family caregivers. Nursing Ethics 20, 748-761.

Oosterveld-Vlug MG, Pasman HR, van Gennip IE, Muller MT, Willems DL and OnwuteakaPhilipsen BD (2014) Dignity and the factors that influence it according to nursing home residents: a qualitative interview study. Journal of Advanced Nursing 70, 97-106.

Oosterveld-Vlug MG, Pasman HRW, van Gennip IE, Willems DL and Onwuteaka-Philipsen BD (2013) Changes in the personal dignity of nursing home residents: a longitudinal qualitative interview study. PLOS ONE 8, e73822. 
Pace R, Pluye P, Bartlett G, Macaulay AC, Salsberg J, Jagosh J and Seller R (2012) Testing the reliability and efficiency of the pilot Mixed Methods Appraisal Tool (MMAT) for systematic mixed studies review. International Journal of Nursing Studies 49, 47-53.

Palacios-Ceña D, Losa-Iglesias ME, Cachón-Pérez JM, Gómez-Pérez D, Gómez-Calero C and Fernández-de-las-Peñas C (2013) Is the mealtime experience in nursing homes understood? A qualitative study. Geriatrics \& Gerontology International 13, 482-489.

Råholm M-B, Lillestø B, Lohne V, Caspari S, Sæteren B, Heggestad AKT, Aasgaard T, Lindwall L, Rehnsfeldt A, Høy B, Slettebø Å and Nåden D (2014) Perspectives of dignity of residents living in nursing homes: experiences of family caregivers. International Journal for Human Caring 18, 34-39.

Ryan RM and Deci EL (2000) Self-determination theory and the facilitation of intrinsic motivation, social development, and well-being. American Psychologist 55, 68-78.

Sæteren B, Heggestad AKT, Høy B, Lillestø B, Slettebø Å, Lohne V, Råholm M-B, Caspari S, Rehnsfeldt A, Lindwall L, Aasgaard T and Nåden D (2016) The dialectical movement between deprivation and preservation of a person's life space. Holistic Nursing Practice 30, 139-147.

Sikorska-Simmons E (2006) The effects of organizational policies on resident perceptions of autonomy in assisted living. Journal of Housing for the Elderly 20, 61-77.

van der Meide H, Olthuis G and Leget C (2015) Participating in a world that is out of tune: shadowing an older hospital patient. Medicine, Health Care and Philosophy 18, 577-585.

Verhoeven I and Tonkens E (2013) Talking active citizenship: framing welfare state reform in England and the Netherlands. Social Policy and Society 12, 415-426.

Walent RJ and Kayser-Jones J (2008) Having a voice and being heard: nursing home residents and in-house advocacy. Journal of Gerontological Nursing 34, 34-42.

Walker H and Paliadelis P (2016) Older peoples' experiences of living in a residential aged care facility in Australia. Australasian Journal on Ageing 35, e6-e10.

Cite this article: van Loon J, Luijkx K, Janssen M, de Rooij I, Janssen B (2021). Facilitators and barriers to autonomy: a systematic literature review for older adults with physical impairments, living in residential care facilities. Ageing \& Society 41, 1021-1050. https://doi.org/10.1017/S0144686X19001557 